\title{
Carbon Dot-Based Hybrid Nanogels for Biomedical Applications
}

\author{
Hui Wang*1 and Rongrong Nie $^{2}$ \\ ${ }^{1}$ High Magnetic Field Laboratory, Hefei Institutes of Physical Science, , China \\ ${ }^{2}$ Department of Prosthdontics, Medical School of Nanjing University, China
}

Received: April 26, 2018; Published: May 08, 2018

*Corresponding author: Hui Wang, High Magnetic Field Laboratory, Hefei Institutes of Physical Science, Chinese Academy of Sciences, China

\section{Introduction}

Stimuli-responsive nanogels have achieved great progress in biomedical fields for clinical diagnosis and therapy due to their porous structures, large surface area, good biocompatibility [1]. Recently, much attention has been focused on the synthesis of stimuli-responsive nanogels with optical maker in polymer network, therefore, realizing the combination of biosensing, bioimaging and controlled drug delivery [2]. Such hybrid nanogels have great potential for disease diagnosis and imaging-guided cancer therapy since the fluorescent imaging not only precisely monitor the change of biosignal in vitro and in vivo, but also identify the location of the tumor region and monitor the treatment progress [3].

Carbon dots (CDs), as a new type of carbon nanomaterial, have demonstrated widely biomedical applications because of their small size distribution, good biocompatibility, tunable photoluminescence (PL), excellent photostability and chemical inertness $[4,5]$. Compared to the other optical marker including noble metal, organic dyes and semiconductor quantum dot NPs, CDs not only demonstrate non or lower toxicity and biocompatibility based on their cytotoxic and in vivo toxic evaluation, but also possess hydrophilic functional groups on the surface of CDs [6]. In addition, CDs can be applied as photothermal agent and twophoton fluorescent (TPF) imaging agent due to their strong NIR absorption and upconversion PL property [7]. Thanks to the combination of these key properties of CDs, it is very meaningful to develop CDs-based hybrid nanogels for biosensing and bioimaging and responsive drug delivery to enhance the therapeutic efficacy. In this mini-review, it will summarize our latest developments and applications and addresses the future perspectives of CDs-based hybrid nanogels in the biomedical field.

\section{Synthesis and Characterization}

To ensure the stability of CDs in the hybrid nanogels, the CDs with smaller size distribution should possess reactive functional groups including- $\mathrm{COOH},-\mathrm{OH}$ or/and - $\mathrm{NH} 2$ groups. These functional groups are expected to react with comonomer via secondary forces (e.g., hydrogen bonding, van der Waals forces, or electrostatic interactions). After copolymerization and crosslinking, the CDs will be randomly embedded in the resultant nanogel networks, leading to the effectively immobilization of CDs in the polymer nanogels. Up to now, several methods have been used to prepare to CDs-based hybrid nanogels, such as: microfluidic technology, nonsolvent induced phase separation and CD-induced association of polysaccharides, emulsion/inverse emulsion polymerization and precipitation polymerization. Figure 1 shows a type example for the synthesis of CD-based hybrid nanogel using one-pot precipitation polymerization method (Figure 1) [3]. Schematic illustration of the formation of PEG-chitosan@CD hybrid nanogels. Reproduced from ref. 3 with permission (2018) of the Royal Society of Chemistry.

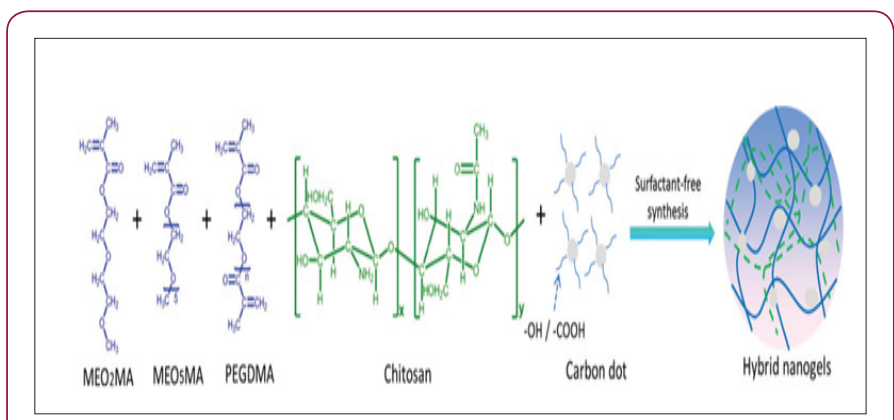

Figure 1: a type example for the synthesis of CD-based hybrid nanogel using one-pot precipitation polymerization method.

Consider the small size and low contrast of CDs, it is difficult to characterize the successful loading of CDs in polymer nanogels. The combination of different measurement may be an alternative strategy to confirm the structure of CDs-based hybrid nanogels. For example, transmission electron microscopy (TEM), high resolution TEM (HRTEM), scanning electron microscopy (SEM), and atomic 
force microscopy (AFM) are useful tools to identify the location and distribution of the CDs in the polymer network. The UV-vis and PL measurement are useful to ensure the stability and optical property of CDs-based hybrid nanogels because the as-obtained hybrid nanogels always undergo the long-term dialysis to remove the free CDs in the hybrid nanogels.

\section{Biomedical Applications}

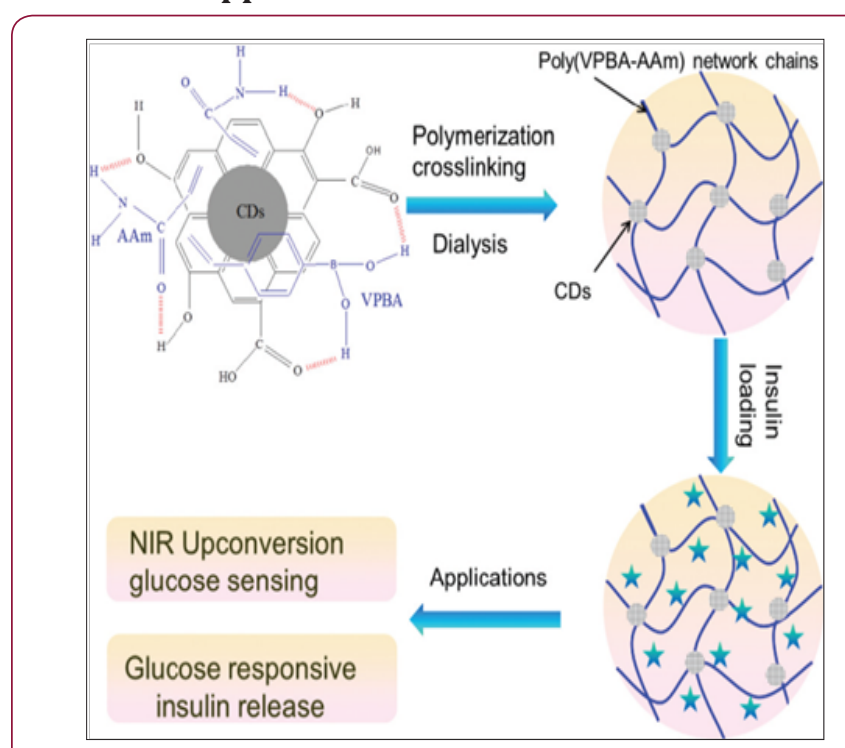

Figure 2.

The combination of responsive nanogels and CDs have widened their applications or enhanced a single property level in different areas of nanomedicine. Nowadays, many CDs-based hybrid nanogels have been designed for biosensing, bioimaging, responsive drug release Wang et al. [8-11]. Reported a CDs-based hybrid nanogels composed of acrylamide (AAm), 4-Vinylphenyl boronic acid (VPBA) and CD for glucose sensing and glucose-responsive insulin release, as shown in Figure 2 [12]. The reversible swelling/ deswelling transition of the poly(VPBA-AAm) nanogels under different glucose concentrations could change the surface states of the CDs and thus affect the fluorescence properties of the hybrid nanogels. The porous property of the poly(VPBA-AAm)-CD hybrid nanogels provides a high loading capacity for insulin molecules and the insulin release rate from the hybrid nanogels can be triggered by the glucose concentration in the dispersion medium. Figure 2 Schematic illustration of multifunctional hybrid microgels with CDs randomly embedded in the glucose-responsive poly(VPBA-AAm) gel network for the integration of NIR upconversion fluorescence glucose sensing and glucose-responsive insulin release. Reprinted from ref. 12 with permission (2016) of the Royal Society of Chemistry.

One example about imaging-guided cancer therapy is the synthesis of the chitosan@CD hybrid nanogels, as shown in Figure 3 [13]. The DOX-loaded chitosan@CD hybrid nanogels demonstrated dual-responsive drug release under NIR light and different $\mathrm{pH}$. In addition, the NIR imaging function of the CD in hybrid nanogels could be applied to monitor the therapeutic effect of tumor by imaging-guided chemotherapy in vivo. More importantly, the therapeu- tic capacity achieved by combined chemo-photothermal therapy is much higher than the additive result from separated photothermal therapy and chemotherapy based on the tumor growth curves, indicating a synergistic effect by NIR-induced cancer therapy. Figure 3 Schematic depiction of DOX-loaded CCHNs for therapeutic application. Reprinted from ref. 13 with permission (2017) of the American Chemical Society.

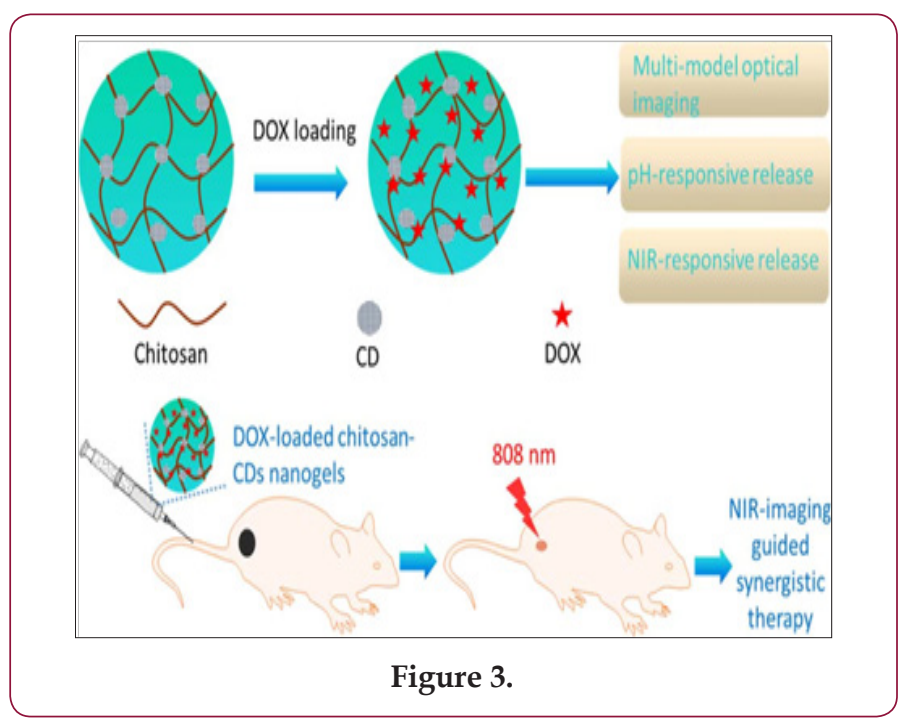

\section{Conclusion}

In summary, we have reviewed the preparation and structure and application of CDs-based hybrid nanogels. Despite the significant advances have been obtained, the clinical translation of CDs-based hybrid nanogels for all other diverse applications remain to be realized. A number of parameters affect the efficacy of this sensing, imaging and drug delivery and requires further optimization, such as: fluorescence emission range, quantum yield and tumor accumulation of hybrid nanogels. The application of these CDs-based hybrid nanogels in the industry is also still in its first steps and there is a strict need for relevant clinical data relating to safety and efficacy of hybrid nanogels in vivo.

\section{References}

1. Molina M, Asadian-Birjand M, Balach J, Bergueiro J, Miceli E, et al. (2015) Stimuli-responsive nanogel composites and their application in nanomedicine. Chemical Society Reviews 44: 6161-6186.

2. Wu W, Zhou S (2010) Hybrid micro-/nanogels for optical sensing and intracellular imaging. Nano Reviews 1: 5730.

3. Wang H, Chen Q Zhou S (2018) Carbon-based hybrid nanogels: a synergistic nanoplatform for combined biosensing, bioimaging, and responsive drug delivery. Chemical Society Reviews.

4. Wang H, Zhuang J, Velado D, Wei Z, Matsui H, et al. (2015) Near-Infraredand Visible-Light-Enhanced Metal-Free Catalytic Degradation of Organic Pollutants over Carbon-Dot-Based Carbocatalysts Synthesized from Biomass. ACS Appl. Mater. Interfaces 7(50): 27703-27712.

5. Lim SY, Shen W, Gao Z (2015) Carbon quantum dots and their applications. Chemical Society Reviews 44: 362-381.

6. Zuo P, Lu X, Sun Z, Guo Y, He H (2016) A review on syntheses, properties, characterization and bioanalytical applications of fluorescent carbon dots. Microchimica Acta 183: 519-542.

7. Sun X, Lei Y (2017) Fluorescent carbon dots and their sensing applications. TrAC Trends in Analytical Chemistry 89: 163-180. 
8. Wang H, Di J, Sun Y, Fu J, Wei Z, et al. (2015) Biocompatible PEGChitosan@Carbon Dots Hybrid Nanogels for Two-Photon Fluorescence Imaging, Near-Infrared Light/pH Dual-Responsive Drug Carrier, and Synergistic Therapy. Advanced Functional Materials 25: 5537-5547.

9. Wang H, Ke F, Mararenko A, Wei Z, Banerjee P, et al. (2014) Responsive polymer-fluorescent carbon nanoparticle hybrid nanogels for optical temperature sensing, near-infrared light-responsive drug release, and tumor cell imaging. Nanoscale 6: 7443-7452.

10. Wang H, Yi J, Velado D, Yu Y, Zhou S (2015) Immobilization of Carbon Dots in Molecularly Imprinted Microgels for Optical Sensing of Glucose at Physiological pH. ACS Applied Materials \& Interfaces 7: 15735-15745.
11. Wang H, Yi J, Mukherjee S, Banerjee P, Zhou S (2014) Magnetic/NIRthermally responsive hybrid nanogels for optical temperature sensing, tumor cell imaging and triggered drug release. Nanoscale 6:1300113011.

12. Wang H, Yi J, Yu Y, Zhou S (2017) NIR upconversion fluorescence glucose sensing and glucose-responsive insulin release of carbon dotimmobilized hybrid microgels at physiological pH. Nanoscale 9:509-516.

13. Wang H, Mukherjee S, Yi J, Banerjee P, Chen Q et al. (2017) Biocompatible Chitosan-Carbon Dot Hybrid Nanogels for NIR-Imaging-Guided Synergistic Photothermal-Chemo Therapy. ACS Appl Mater Interfaces 9: 18639-18649.

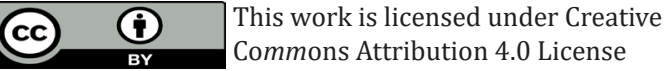

Submission Link: https://biomedres.us/submit-manuscript.php

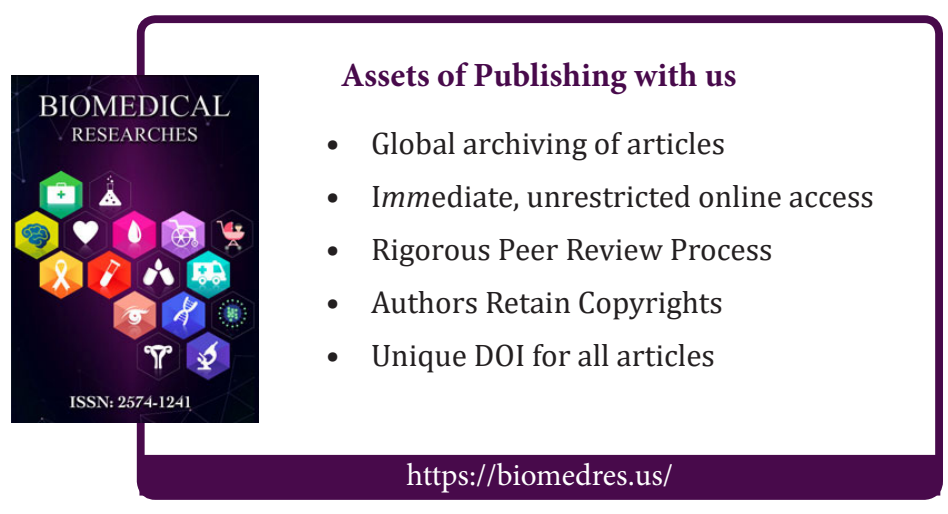

